\title{
Update on Chronic Rejection After Intestinal Transplant: An Overview From Experimental Settings to Clinical Outcomes
}

\author{
Augusto Lauro, ${ }^{1}$ Ignazio R. Marino ${ }^{2}$
}

\begin{abstract}
Chronic rejection affects the long-term survival of solid-organ transplants, accounting for an incidence of between $5 \%$ and $10 \%$ after intestinal/multivisceral transplant. Because of unclear symptoms and signs and endoscopic findings, the diagnosis is often delayed. Presently, allograft removal represents the only available therapy due to the absence of effective pharmacologic approaches. Extensive research, through animal models, has been performed over the past $\mathbf{2 0}$ years to clarify the complex immune- and nonimmune-mediated mechanisms behind the development of chronic allograft enteropathy, with the aim of elucidating how to avert chronic rejection. The role of donor-specific antibodies and the way to challenge them in the clinic have gained acceptance among transplant centers as one of the main steps to prevent chronic rejection, although no common protocol exists that can be applied in a systematic fashion. The adjunct of a liver graft when retransplanting is needed in a sensitized recipient due to its protective effect against humoral immunity. Multicenter studies and clinical trials are required to better understand the pathogenesis of chronic rejection and to find the therapeutic answer to this clinical query.
\end{abstract}

Key words: Biopsy, Chronic allograft enteropathy, Donorspecific antibodies, Immunosuppression

\section{Introduction}

Since publication of studies from the Pittsburgh group dating from the early 1990s, ${ }^{1-4}$ chronic rejection

From the St. Orsola University Hospital Alma Mater Studiorum, Bologna, Italy; and the ${ }^{2}$ Sidney Kimmel Medical College, Thomas Jefferson University, Philadelphia, Pennsylvania, USA

Acknowledgements: The authors received no financial support and have no conflicts of interes to report for this study. We thank Ms. Claudia Cirillo for English language editing of our text. Corresponding author: Augusto Lauro, St. Orsola University Hospital, Alma Mater Studiorum, Bologna, Italy

Phone:+39051 2143721 E-mail: augusto.lauro@aosp.bo.it

Experimental and Clinical Transplantation (2019) 1: 18-30
(CR) has been viewed as the major cause of late graft loss and reduced late patient survival after intestinal/multivisceral transplant (ITx). This phenomenon has not been changed by recent immunosuppressive preconditioning protocols. ${ }^{5}$ It presents as enteropathy, which has an incidence ranging from $10 \%$ to $20 \%$ in historical series, ${ }^{6,7}$ and follows an insidious, progressive course lacking early or specific clinical symptoms or mucosal findings through endoscopy. The median time to development of CR is 39 months, with a range of 22 to 67 months. ${ }^{7}$ The risk increases 2 years after transplant, achieving a nadir during posttransplant year 3 . An isolated small bowel transplant appears to render the graft more susceptible to $\mathrm{CR}$ than liver-intestine transplant procedures $(21 \%$ vs $5 \%){ }^{7}$ A consensus conference (unpublished results) held in June 2015 in Buenos Aires, Argentina during the XIV Intestinal Small Bowel Transplant Symposium (ISBTS; now named Congress of Intestinal Rehabilitation and Transplantation Association) proposed several criteria to identify CR: recipients can present with abdominal pain, mass, or distension with chronic diarrhea, bowel obstruction, enterocutaneous fistulas, intolerance to feeding with recurrent emesis, weight loss, enteropathy with protein loss, failure to thrive, or complications of ostomy site. Similar to acute cellular rejection (ACR), reliable fecal and serum markers are not available. The conference proposed decreased citrullin (concomitant loss of graft mass and function) and elevated C-reactive protein/lipopolysaccharide-binding protein (mucosal translocation) as surrogate CR markers.

Computed tomography and magnetic resonance imaging may support diagnosis by showing thickening of mesentery and/or intestinal wall and rarity of mesenteric vessels. Close endoscopic monitoring is mandatory for all ITx recipients: the common endoscopic presentation of $\mathrm{CR}$ is persistent, 
non-healing, focal mucosal ulceration, which is often preceded by repeated episodes of ACR. ${ }^{8}$ Later, mucosal folds become effaced with pseudomembranes, and the bowel appears firm and fibrotic. Biopsy specimens may show mild ischemic changes, low-grade apoptosis, crypt loss, and often mild fibrosis of the lamina propria. ${ }^{8}$ Mucosal biopsies are often noncontributory, even in front of refractory intestinal dysfunction. In biopsies with fibrosis, the cause may be unclear because fibrosis may be secondary to other causes, such as previous episodes of rejection, ischemic injury, prior infections, medication-associated chronic injury, and prior biopsy site irritation. ${ }^{8}$ Thus, clinical and endoscopic diagnoses of $C R$ are difficult.

Chronic rejection is characterized by an arteriopathy with blunting of villi, concentric vasculopathy, luminal occlusion, leukocyte infiltration, and a marked fibrotic change. ${ }^{6}$ The marked intimal hyperplasia associated with fibrosis leads to impaired vascular perfusion of the graft. ${ }^{8}$ Unfortunately, this finding is seen in submucosal or mesenteric arteries, which are not normally sampled on an endoscopic biopsy.

There is much discussion among transplant centers on "when" to perform graft explants in cases of irreversible chronic allograft enteropathy (that is, prior or during retransplant), and many pro and con arguments were debated during the already quoted ISBTS 2015 meeting (unpublished results). Nevertheless, diagnosis of CR is usually confirmed only in full-thickness biopsies through explanted failed grafts that show the associated obliterative vasculopathy. ${ }^{8}$ Studies and discussion on this issue continue. Therefore, it is worthwhile to review the experimental findings and clinical series on this topic to get a wider picture, to clarify the updated knowledge, and to increase the relative awareness in the medical community.

Pathogenesis from acute cellular rejection to chronic rejection: The role of immunosuppression The relationships between ACR (a recurring occurrence after ITx) and CR and their correlations with immunosuppressive treatment require clarification. Although the frequency of ACR has decreased from historic levels of $80 \%$ to present levels of $20 \%$ to $40 \%$ in the early phase, ${ }^{5}$ rejection episodes remain one of the most frequent complications after ITx. Occurrence of severe ACR within 30 days posttransplant, a higher number of ACR episodes, and late-onset ACR have been associated with progression to CR. ${ }^{9}$ Although adequate control of ACR in the early phase has resulted in improved short-term graft and patient survival, the impact of recurrent ACR episodes on the development of $\mathrm{CR}$ graft dysfunction and the recovery after ACR are not well understood.

Intestinal transplant models of long-term $\mathrm{CR}$ in the allograft have been used to elucidate its mechanisms. In 2000, de Bruin and associates ${ }^{10}$ performed experimental ITx through a Dark Agoutito-AS rat strain combination. Rats received shortterm (days -2 to +9 ) or long-term (until day 50) intramuscular cyclosporine $(5 \mathrm{mg} / \mathrm{kg})$ to prevent ACR. Controls were untreated allografts, Dark Agouti isografts with and without cyclosporine, and normal Dark Agouti and AS rats. Rats were followed for 50 and 100 days after transplant. Animals that survived had histologic signs of CR by 50 and 100 days after transplant; however, long-term cyclosporine treatment delayed its development, with functional abnormalities only seen in animals that were treated with short-term therapy.

In 2013, Li and colleagues ${ }^{11}$ used Fisher 344 and Lewis rats as ITx donors and recipients, respectively. Animals received intramuscular cyclosporine (5 $\mathrm{mg} / \mathrm{kg} /$ day) from postoperative day (POD) 0 to POD13 and intramuscular FK506 $(0.3,0.5$, or $1.0 \mathrm{mg} / \mathrm{kg} /$ day) on POD0 to POD13, POD20, and POD27. In the cyclosporine group, all allografts demonstrated one or more histologic features of $\mathrm{CR}$ but no serious villous blunting. In addition, there were no significant difference in features of $C R$ between POD60 and POD90. In the FK506 group, the rats that received FK506 at doses of 0.3 or $0.5 \mathrm{mg} / \mathrm{kg} /$ day survived to POD126 but died before POD156, whereas recipients that were administered FK506 at $1.0 \mathrm{mg} / \mathrm{kg} /$ day survived beyond POD180 and showed the same body weight gain as the isogeneic group. Histopathologic analyses revealed distinctive features of $\mathrm{CR}$ in the rat models induced by cyclosporine or FK506. The authors concluded that recipients administered FK506 survived longer but showed more classic characteristics of CR than those treated with cyclosporine.

These 2 experimental studies showed that histologic findings of $\mathrm{CR}$ are the final results of a complex, multistage process of cellular injury and inflammation, where repetitive insults exhaust the recipient's natural repair mechanisms, leading to fibrotic replacement and intestinal failure. Emerging 
evidence suggests that immune-mediated injuries to the graft are the fundamental cause of CR. ${ }^{12}$

\section{Pathogenesis of donor-specific antibodies and chronic rejection}

Recently, the role of humoral alloimmunity has also appeared to be closely related to CR after solid-organ transplant, and the presence of circulating donorspecific antibodies (DSA) may be associated with worse clinical outcomes after ITx as well. The major target of humoral immunity appears to be the graft endothelium, which can be activated and injured by HLA antibodies, but the mechanisms by which humoral alloimmunity leads to CR are not clear.

Several trials have been conducted to verify the relationship between CR and DSA in solid-organ transplant. In the field of ITx, the first clinical report came in 2000 from Pittsburgh, ${ }^{13}$ in which $18 \%$ of recipients of ITx harbored preformed antidonor immunoglobulin G lymphocytotoxic antibodies in sera. Compared with other solid-organ transplant recipients, Bond and colleagues ${ }^{13}$ attributed this relatively high rate of preformed antibodies to the multiple abdominal operations that these patients receive before transplant, together with the frequent need for numerous blood transfusions. Similar to other solid-organ allografts, positive T-cell lymphocytotoxic crossmatch increased the frequency and severity of intestinal rejection. Such a risk was significantly higher among patients who received ITx only, whereas those who also had simultaneous liver transplant (as part of a composite visceral allograft) seemed to ameliorate the negative effects of the preformed antibodies and crossmatch reactivity.

Twelve years later, Abu-Elmagd and associates ${ }^{14}$ from the same group performed a retrospective study to investigate the effects of HLA antibodies on CR among 194 consecutive primary adult ITx recipients. Of these patients, $71(37 \%)$ also received liver allografts. Immunosuppression was tacrolimus based with antilymphocyte recipient pretreatment in 150 patients $(77 \%)$. The authors observed a relatively high incidence of CR $(19 \% ; 36$ cases) in their series, in which the average follow-up was $21 \pm 10$ months (range, $288 \mathrm{mo}$ ). Crossmatch was positive in 55 patients $(28 \%)$. HLA DSAs were detectable before transplant in 49 recipients (31\%), with 19 continuing to have circulating antibodies. Another 19 recipients (18\%) developed de novo DSA. Cumulative risk of $\mathrm{CR}$ was slightly higher in recipients with a positive crossmatch versus a negative crossmatch. Presence of persistent and de novo HLA DSAs significantly $(P<.001)$ increased risk of $\mathrm{CR}$ and associated graft loss, and this event was irreversible in one-third of the patients. Inclusion of the liver was a significant predictor of better outcome $(P=.004$, hazard ratio $=$ $0.347)$, with significant clearance of preformed antibodies $(P=.04$, odds ratio $=56)$ and lower induction of de novo DSA $(P=.07$, odds ratio $=24)$. Overall, patients who also had liver transplants showed reduced rates of CR. The results reported by Abu-Elmagd and associates illustrate a strong relation between DSA and an increased risk of CR with allograft failure after ITx.

In 2015, Kubal and associates ${ }^{15}$ prospectively screened sera for DSA in 79 recipients up to 3 years after ITx. Standard immunosuppression included thymoglobulin-rituximab induction and tacrolimusprednisone maintenance. Twenty-two patients $(28 \%)$ developed de novo DSA at a median posttransplant period of 3 months (range, 1-36 mo). De novo DSAs were observed in 10 of 40 liver-included and 12 of 39 liver-excluded transplants $(P=.57)$. Chronic rejection $(14 \%$ vs $5 \% ; P=.21)$ and graft loss due to ACR $(18 \%$ vs $7 \% ; P=.14)$ were numerically higher in patients with de novo DSA. In their series, de novo DSA formation, particularly early in the posttransplant course, was associated with a trend toward worse outcomes.

Both of the above studies were arguments in favor of the use of "virtual" crossmatch before ITx to prevent this phenomenon. However, plasmapheresis and rituximab may also reduce the concentration of circulating HLA antibodies, even if both methods are ineffective against antibody-producing plasma cells, which belong to the bone marrow compartment. Consequently, splenic and bone marrow plasma cells do not respond to typical desensitization regimens: proteasome inhibitors like bortezomib deplete nonmalignant plasma cells and suppress T-cell function, providing effective treatment of antibodymediated rejection. ${ }^{16}$

Few reports have shown evidence of bortezomib efficacy in the reduction and elimination of DSAs after renal, pancreas, and intestinal transplant. In an unpublished report from 2011 at the XII ISBTS meeting (Washington, DC, USA), the Pittsburgh group presented a small series of 5 recipients treated by bortezomib for DSA positivity. Likewise, in 2011, Gerlach and associates ${ }^{16}$ reported on patients affected 
by recurrent ACR and successfully treated by bortezomib. At the end of the treatment, anti-HLA antibodies were almost entirely eliminated, and there was a complete resolution of inflammatory graft alteration under tacrolimus (trough levels of 4-5 ng/mL) and mycophenolate mofetil $(2 \times 1000$ $\mathrm{mg}$ ). As a result of persistent DSA levels despite exhaustive treatment, histologic signs of chronic allograft alterations (ie, fibrosis) showed CR in the allograft.

The above studies suggest that DSA development after ITx is associated with CR and that high numbers of antigen and epitope mismatches between donors and recipients represent significant risk factors for DSA development. Antibody-mediated rejection may be accepted as an entity of "vascular" rejection, and persistently high DSA levels may continuously injure the graft, yielding risk of treatment-refractory allograft rejection.

\section{Pathogenesis of the "liver protective effect" on chronic rejection}

In 2000, a rat model was used by Meyer and associates ${ }^{17}$ to investigate the tolerogenic effects of a liver allograft simultaneously transplanted with a small bowel. The group compared a rat model of isolated ITx versus the combined model, in which syngeneic Lewis and 2 fully allogeneic rat strain combinations (Brown Norway-to-Lewis and Dark Agouti-to-Lewis) were used. Clinical course and histologic findings after isolated ITx demonstrated $\mathrm{CR}$ of the allograft within 100 days. In the liver plus small bowel transplant model, long-term (> 150 days) graft acceptance was achieved, although initial immunosuppression was significantly lower.

To confirm the role of the liver in clearing DSAs in highly sensitized recipients, which could thus reduce $\mathrm{CR}, \mathrm{Wu}$ and $\mathrm{Cruz}^{18}$ analyzed outcomes of retransplant procedures over 10 years, comparing 13 adult patients who received liver-free retransplants versus 10 patients who received liver-inclusive retransplants. Patient survival rates at 1, 3, and 5 years were $91.7 \%, 55.6 \%$, and $41.7 \%$, respectively, in those with liver-free retransplant compared with $90.0 \%, 80.0 \%$, and $80.0 \%$ in patients with liverinclusive retransplant. Graft survival rates at 1,3, and 5 years in liver-free retransplants were $76.2 \%, 40.6 \%$, and $27.1 \%$, respectively, which were significantly worse than those in liver-inclusive retransplants $(P=.03)$. Within an average follow-up of 32.3 months,
8 of 13 patients $(61.5 \%)$ with liver-free retransplant underwent enterectomy because of severe ACR $(n=3)$ or $\mathrm{CR}(\mathrm{n}=5)$, and 6 of 13 recipients $(46.2 \%)$ succumbed to rejection-related complications. Compared with liver-free retransplants, the rate and severity of ACR were markedly decreased in liver-inclusive retransplants, and no incidence of CR was seen. Within an average follow-up of 44.5 months, 2 of 10 recipients $(20 \%)$ died due to graft-versus-host disease (GVHD) and infection, respectively. The investigators concluded that including the liver as part of an intestinal graft should be considered in adult recipients when retransplant becomes necessary.

\section{Endoscopic/bioptic diagnosis of chronic rejection: mucosal fibrosis}

Independently from pathogenesis of CR, its diagnosis has always been difficult due to lack of clear clinical symptoms or signs and the vagueness of endoscopic/ biopsy findings. In 2006, the presence of mucosal fibrosis in graft biopsies as indicative of $\mathrm{CR}$ was investigated in 182 human ITx recipients. ${ }^{19}$ Kaplan-Meier analysis showed that, within 5 years posttransplant, $33 \%$ of ITx patients had graft biopsies positive for mucosal fibrosis. Although the presence of mucosal fibrosis did not affect patient or graft survival, patients with this lesion were at higher risk of developing chronic allograft enteropathy.

In 2013, Swanson and associates ${ }^{20}$ suggested that some mucosal alterations of CR, seen through biopsy, could correlate with histologic diagnosis in specimens. The group retrospectively investigated explanted human small bowel allografts for histologic alterations in the mucosa and submucosa, showing that crypt epithelial mucin loss and submucosal fibrosis were statistically associated with CR.

From an experimental point of view, Kouwenhoven and associates ${ }^{21}$ reported that the cytokine basic fibroblast growth factor (bFGF) might be involved in tissue remodeling of $\mathrm{CR}$, causing the fibrotic changes. They performed ITx in an allogeneic Dark Agouti-to-AS rat combination and administered cyclosporine to prevent acute rejection. Controls were Dark Agouti isografts and normal Dark Agouti. The group evaluated $b F G F$ gene expression using reverse transcriptase polymerase chain reaction, determining bFGF protein by immunohistochemistry. Allografts showed histologic features of $C R$, whereas isografts preserved normal architecture; bFGF gene expression was present in normal ileum and significantly 
upregulated in allografts. Immunohistochemical staining showed a significant increase in bFGF protein compared with isografts. Most bFGF-positive cells were localized in the submucosa and muscularis, particularly around the neural plexus, and appeared to be activated macrophages.

In a more recent study, Pech and associates ${ }^{9}$ investigated the process of intestinal recovery after ACR and potential mechanisms for functional graft deterioration, possibly inducing $\mathrm{CR}$, in a major histocompatibility complex (MHC)-disparate rat strain combination (Brown Norway to Lewis). Two rescue therapies (FK506 monotherapy and FK506 plus infliximab) were efficacious in reversing ACR in the ITx rodent model. Interestingly, not all parameters returned to normal during the observation periods (14 and 21 days after ITx), which was characterized by $\mathrm{ACR}$ and subsequent rescue therapy. In particular, the authors showed a remarkable increase of fibrosis after ACR and rescue therapy with a concomitant increase of the fibrogenic mediator vascular endothelial growth factor, even as early as 2 and 3 weeks after ITx in both rescue groups. In this experimental setting, reversal of ACR resulted in early-stage graft fibrosis, clearly demonstrating increased graft fibrosis after treatment and recovery from ACR, even after only 1 episode of rejection.

\section{Microchimerism and tolerance to prevent chronic rejection}

Bone marrow chimerism has been successful in inducing tolerance in ACR and CR models after heart and kidney transplant. At the beginning of 2000, the highly immunogenic small bowel allograft provided a rigorous test of this tolerance regimen in many experimental settings. In 2000, Orloff and associates ${ }^{22}$ examined whether induction of tolerance by bone marrow chimerism could prevent vascular sclerosis (VS), typical of CR, in a model of Fisher 344 to Lewis rat ITx. Bone marrow chimeras (BMC) were created by transplant of bone marrow T-cell-depleted Fisher 344 rats into irradiated Lewis rats. Chimerism was assessed by flow cytometry. The Fisher 344 model, heterotopically transplanted into the chimeras, was clinically and histologically assessed for CR. Fisher 344 grafts, transplanted into cyclosporine-treated Lewis recipients, served as control grafts for CR. Cyclosporine-treated Lewis rats chronically rejected the Fisher 344 grafts. In contrast, the BMC group demonstrated tolerance and had long-term graft survival (> 120 days) without VS. The BMC group demonstrated immune competence by prompt rejection of third-party allografts in ACI (RT1av1) rats. Bone marrow chimerism prevented chronic graft failure secondary to VS in a model of CR. Vascular sclerosis failed to develop when tolerance was established, suggesting that the mechanisms involved in VS are, in part, immunologically mediated.

Tolerance-inducing strategies that infuse donor bone marrow cells in conjunction with costimulation blockade have also been applied experimentally. ${ }^{23}$ Transplanted intestines from BALB/c mice into C57BL / 6 recipients were treated with anti-CD40L monoclonal antibody, CTLA4 immunoglobulin, donor bone marrow, and busulfan. After completion of this regimen, most transplanted mice developed hematopoietic macrochimerism (although the degree of chimerism varied widely between recipients) and experienced long-term allograft survival. $T$ cells from these mice showed donorspecific hyporesponsiveness in vitro. However, $\mathrm{T}$ cells from chimeric mice proliferated to donor alloantigen in vivo. Furthermore, chimeric mice bearing intestinal allografts were capable of rejecting subsequently placed donor-strain skin grafts. These data suggest that, although long-term allograft survival occurs in the absence of ACR or CR, recipient mice are not completely unresponsive to donor alloantigens. When ITx was performed at the time of initial bone marrow infusion (initiation of the chimerism protocol), most recipients failed to develop chimerism and promptly rejected the intestinal allograft.

Murase and associates ${ }^{24}$ hypothesized that infusion of donor bone marrow cells (BMCs) in recipients of irradiated intestine would improve tolerogenesis without increasing the risk of GVHD. The passenger leukocytes in the intestine have a lineage profile that predisposes to GVHD in some animal models and have inferior tolerogenic qualities compared with leukocytes in the liver, other solid organs, and bone marrow. Ex vivo irradiation of mature lymphoid elements from bowel allografts is known to eliminate the GVHD risk. The authors performed ITx in a GVHD-prone Lewis-to-Brown Norway combination and the reverse GVHDresistant Brown Norway-to-Lewis model under a short course of tacrolimus treatment $(1 \mathrm{mg} / \mathrm{kg} /$ day on days $0-13,20$, and 27). Grafts were irradiated ex 
vivo using a 137Cs source. In selected experimental groups, donor BMCs $(2.5 \times 108)$ were infused on the day of ITx. The unmodified Lewis intestine remained intact (whether transplanted alone or with adjunct donor BMC infusion), but all the Brown Norway recipients died of GVHD after approximately 2 months. Intestinal graft irradiation (10 Gy) effectively prevented GVHD and prolonged survival to 92.5 days, but all Brown Norway recipients died with CR of the Lewis grafts, which was prevented by infusion of adjunct donor BMCs without causing GVHD. In the GVHD-resistant reverse strain direction (Brown Norway-to-Lewis combination), all ITx recipients treated for 27 days with tacrolimus survived $\geq 150$ days without regard for graft irradiation or adjunct BMC. However, CR was severe in the irradiated intestine, moderate in the unaltered graft, and least in the irradiated intestine transplanted with adjunct BMC. Mild arteritis in the 150-day allografts of both strain combinations may have been associated with irradiation, but this was prevented when weekly doses of tacrolimus were continued for the duration of the experiment rather than being stopped at 27 days. The authors concluded that recipients were protected from GVHD by irradiating intestinal allografts, but the resulting leukocyte depletion led to $\mathrm{CR}$ of the transplanted bowel. Adjunct donor BMC prevented CR without causing GVHD.

To examine whether long-term graft function is associated with donor-specific tolerance, Timmermann and associates ${ }^{25}$ analyzed the functional status of recipient $\mathrm{T}$ cells in vivo and in vitro. One-step orthotopic ITx was performed in the allogeneic Brown Norway-to-Lewis rat strain combination. FK506 was given daily at a dose of $2 \mathrm{mg} / \mathrm{kg}$ from days 0 to 5 in the rejection model and from days 0 to 9 in the long-term functional model. Mean survival time in the rejection model was $98 \pm 2.8$ days. Histologic examination of these small bowel allografts disclosed signs of CR. In contrast, all longterm functional animals survived for $>250$ days without clinical signs of CR. Furthermore, this animal model showed evidence of donor-specific tolerance, whereas heterotopic Dark Agouti hearts were rejected regularly within 7 days and Brown Norway hearts survived indefinitely ( $>70$ days). In vitro, mixed leukocyte reactivity of CD4-positive $\mathrm{T}$ cells was similarly strong against donor (Brown Norway) antigens as against third-party (Dark Agouti) antigens. The split tolerance, shown by in vivo and in vitro results, enabled acceptance of both the donor-specific heart allografts and small bowel allografts without signs of CR.

In 2016, Ceulemans and associates ${ }^{26}$ translated the Leuven immunomodulatory protocol (LIP) from the laboratory to the clinic. This protocol consisted of donor-specific blood transfusion, avoiding high-dose steroids and calcineurin inhibitors and minimizing reperfusion injury/endotoxin translocation, to promote T-regulatory cell (Treg)-dependent graftprotective mechanisms. Regulatory $\mathrm{T}$ cells may promote the establishment of allogeneic mixed chimerism and the induction of donor-specific tolerance, controlling both ACR and $\mathrm{CR}$ and contributing to protection of the allograft from ischemia-reperfusion injury (IRI). The LIP was tested by the authors in 13 consecutive recipients of deceased ITx donations over 15 years. Early ACR developed in 2 recipients $(15 \%)$, and late ACR developed in 3 recipients $(23 \%)$; all were reversible. There was no occurrence of CR. At last follow-up (3.5 y; range, 0.5-12.5 y), no DSAs were detected. However, a high frequency of circulating CD4+CD45RA-Foxp3hi memory Tregs was found $(1.8 \%$; range, $1.39 \%$ to $2.21 \%$ ), comparable to that shown in tolerant kidney transplant recipients and superior to stable immunosuppressed kidney transplant recipients, kidney transplant recipients with $C R$, and healthy volunteers. In this human ITx cohort, the authors showed that donor-specific blood transfusion in a lowinflammatory/proregulatory environment activates Tregs at levels similar to tolerant kidney transplant patients without causing sensitization. In their setting, LIP limited ACR and CR under reduced immunosuppression and thereby prolonged longterm survival after ITx.

\section{Fish oil and chronic rejection: did we find the "holy grail"?}

Fish oil has been long debated as beneficial to $C R$ since the beginning of the new millennium. Recently, Wang and associates ${ }^{27}$ investigated the beneficial effects of $n-3$ polyunsaturated fatty acids (n-3 PUFA) enriched with fish oil on CR. Laboratory animals were divided into 3 groups: the isograft group (cyclosporine + corn oil-supplemented diet), the allograft group (cyclosporine + corn oil-supplemented diet), and the allograft-2 group (cyclosporine + fish oil-supplemented diet). Donor intestines from Fisher 344 rats were transplanted orthotopically into Lewis 
rat recipients. Cyclosporine was administered at $5 \mathrm{mg} / \mathrm{kg} /$ day for 2 weeks postoperatively. Chronic rejection developed on POD90, but n-3 PUFA significantly decreased CR scores and increased postoperative weight gain. This attenuation was associated with reduced graft interleukin 15 expression, a key player in various immunologic processes, including CR after renal and cardiac transplant. Fish oil contributed to improved pathologic and clinical outcomes with regard to chronic small bowel allograft rejection, and this improvement was associated with reduced interleukin 15 graft expression.

$\mathrm{Li}$ and associates ${ }^{28}$ also examined the role of fish oil in the post-CR recovery of gut microbiota. With the use of denaturing gradient gel electrophoresis at 190 days after ITx, the authors showed the luminal and mucosal microbiota composition in CR rats by sequence analyses. Furthermore, changes in the localization of intestinal tight junction (TJ) proteins were examined by immunofluorescent staining. These analyses revealed that gut microbiota in CR rats had a shift toward increased Escherichia coli, Bacteroides species, and Clostridium species and a decreased abundance of Lactobacillus bacteria in the intestine. Fish oil supplementation enhanced the recovery of gut microbiota, showing a significant decrease of gut bacterial proportions of Escherichia coli and Bacteroides species and an increase of Lactobacillus species. In addition, CR rats showed pronounced alteration of TJs, depicted by marked changes in epithelial cell ultrastructure and redistribution of the $\mathrm{TJ}$ proteins occludens and claudin, as well as disruption in TJ barrier function. Fish oil administration ameliorated disruption of epithelial integrity in CR, which was associated with improved mucosal structure, leading to recovered TJs. This study presented evidence that fish oil is involved in the maintenance of epithelial TJ integrity and recovery of gut microbiota, which may have therapeutic potential against CR after ITx.

In 2003, Wei and associates ${ }^{29}$ investigated the role of n-3 PUFA in preventing chronic allograft vasculopathy $(\mathrm{CAV})$, a typical feature of CR after ITx. Fish oil potentially regulates the pathway mediated by the receptor for advanced glycation end products (RAGE) and its ligand high mobility group box-1 (HMGB1), which may contribute to the pathogenesis of CAV. Using a CR model of rat ITx, the group investigated whether amelioration of $\mathrm{CAV}$ by fish oil feeding was associated with regulation of the RAGE signaling pathway. The authors administered lowdose fish oil to rats for 190 postoperative days. Male inbred Lewis rats and Fisher 344 rats were used to establish a CR model of ITx. Recipient rats received phosphate-buffered saline or fish oil at a daily dose of $3 \mathrm{~mL} / \mathrm{kg}$. All rats were alive on POD190. The expression of HMGB1 and RAGE increased in CAVbearing vessels. Feeding low-dose fish oil for 6 months attenuated CAV and significantly reduced HMGB1 and RAGE expression levels, indicating that the beneficial effects of low-dose fish oil on CAV may occur via down-regulation of the HMGB1-RAGE pathway.

In contrast, a 2012 study by Wei and associates ${ }^{30}$ showed that feeding of corn oil containing low levels of n-3 PUFA had no favorable effects on CAV development and failed to decrease HMGB1 and RAGE expression. With regard to clinical settings, there are no studies in the English literature reporting on the role of fish oil in human small bowel CR after ITx.

Matsumoto and colleagues ${ }^{31}$ debated its role on total parenteral nutrition liver disease, comparing hepatic explant pathology and biochemical outcomes of pediatric patients with intestinal failure who had received a liver-inclusive ITx. Their aim was to evaluate the effects of parenteral fish oil lipid emulsion on cholestasis and fibrosis of explanted livers compared with standard omega-6 polyunsaturated fatty acid (O6FA) parenteral nutrition after ITx. Seven liver-inclusive ITxs were performed in 7 patients who received fish oil for a mean of $62 \% \pm 13 \%$ of total patient lifespan (16.1 $\pm 7.0 \mathrm{mo})$ before transplant. Median total bilirubin fell significantly from $6.9 \mathrm{mg} / \mathrm{dL}$ at the start of treatment to $0.7 \mathrm{mg} / \mathrm{dL}$ at the time of transplant $(P<.02)$ compared with the similarly matched O6FA cohort $(P=.012)$. All 7 explanted livers from fish oil-treated intestinal recipients had advanced fibrosis (stage 3 or 4 ) noted on explant pathologic examination, despite resolution of cholestasis at the time of transplant. Histologic inflammatory scores were lower $(P=.056)$ in the fish oil group, with similar degrees of advanced fibrosis as in the O6FA group. Their conclusion was that significant hepatic fibrosis was present in the explanted livers despite a reduction in inflammation, confirming concern that the use of fish oil may have a limited role in altering the development of hepatic fibrosis from parenteral nutrition. 
Long-term survival and chronic enteropathy: The role of tumor necrosisfactor $\alpha$ Inhibition and nonHLA antibodies

It has been hypothesized that anti-tumor necrosis factor $\alpha$ (TNF- $\alpha$ ) therapy may help diminish chronic, inflammatory long-term effects after solid-organ transplant, avoiding chronic allograft enteropathy in the ITx field. In a previous report, Pech and associates ${ }^{9}$ studied the effects of anti-TNF- $\square$ therapy in an experimental ITx setting. Reversal of severe ACR by infliximab resulted in early-stage graft fibrosis (an early sign of CR), clearly demonstrating increased fibrosis in the bowel after both treatment and recovery from severe ACR, even after only 1 episode of rejection. Langrehr and associates ${ }^{32}$ used TNF- $\alpha$ inhibition to prolong long-term survival, performing ITx in a Dark Agouti-Lewis rat combination. Recipients were treated perioperatively with either the anti-CD4 antibody RIB5/ 2 (days -1 and 0 and on POD1, POD2, POD4, POD7, POD10, POD14, POD17, and POD21), the anti-TNF antibody etanercept (60 $\mathrm{min}$ before reperfusion and on POD3, POD6, and POD9), or a combination of both. Survival, histology, and expression of immunologic mediator genes on days 3 and 4 after transplant were investigated. Treatment with anti-CD4 antibody alone $(19.71 \pm 5.94)$ and the antibody combination $(171.58 \pm 122.76)$ prolonged survival. The chemokine MIP-1 $\alpha$ was significantly decreased in both anti-CD4 antibody treatment groups, possibly indicating an additional effect of TNF- $\alpha$ blockade on the immune modulation by RIB5/2. Their study demonstrated long-term graft survival with short-term anti-CD4 antibody plus TNF- $\alpha$ antibody treatment in more than $50 \%$ of the recipients of intestinal grafts.

The question of whether the "TNF- $\alpha$ inhibition" effect on the transplanted bowel was always related to ACR reversal was addressed by Gerlach and associates. ${ }^{33}$ Intestinal transplant patients with ileitis/anastomositis who were given infliximab showed no ACR. Two patients with ulcerative distal graft inflammation (resembling terminal ileitis) and inflammation of the ileocolic anastomosis were treated with infliximab even when histologic signs of ACR were not detected. Both patients presented with permanent clinical signs of inflammation (subfebrile temperatures, weight loss), suggesting chronic inflammation and bacterial translocation. Histologic examination showed an ulcer with granulation tissue and fibrinoid necrosis on the surface, with no increase in apoptosis and no infiltration of lymphocytes (no ACR). There was a rapid recovery of clinical signs and laboratory markers after the first infliximab administration ( $5 \mathrm{mg} / \mathrm{kg}$ body weight), achieving sustained remission in 1 patient and transient remission in the other. The authors concluded that late organ impairment with ileal ulceration after ITx may present some form of inflammatory graft enteropathy more similar to inflammatory bowel disease than to rejection, which might be appropriate for long-term TNF- $\alpha$ inhibition versus a standard antirejection regimen.

The same group ${ }^{34}$ also studied the effects of different TNF- $\alpha$ inhibitors on acute IRI and longterm inflammatory responses in experimental ITx. Orthotopic ITx was performed in an isogenic ischemia-reperfusion model of Lewis rats. The TNF- $\alpha$ inhibition groups received either infliximab after reperfusion; etanercept before reperfusion and at POD1, POD3, POD5, and POD7; or pentoxifylline before reperfusion and at POD1 to POD5. Tissue samples were taken from proximal and distal graft sections and mesenteric lymph nodes at 20 minutes, 12 hours, 7 days, and 6 months after reperfusion for histopathology, immune histology, terminal deoxyribosyl transferase-mediated dUTP nick-end labeling, and real-time polymerase chain reaction. Lung sections were stained for the myeloperoxidase assay. In all treatment groups, TNF- $\alpha$ inhibitors decreased inflammation after IRI. Infliximab significantly improved 7-day survival and reduced the histologic and immune histochemical signs of IRI, the numbers of graft-infiltrating T cells, ED1 monocytes, and macrophages, and pulmonary neutrophil infiltration; it also enhanced the accumulation of cytoprotective markers. Graft injury was more prominent in the distal graft than in the proximal graft in all groups, regardless of TNF- $\alpha$ inhibition. Infliximab significantly reduced both acute IRI and, as with other TNF- $\alpha$ inhibitors, the long-term inflammatory response after rat ITx. The author's conclusion was that TNF- $\alpha$ inhibition may help diminish chronic long-term inflammation and avoid chronic allograft enteropathy.

Whether humoral immunity is deeply involved in CR development after ITx and its possible role in this phenomenon was studied in non-HLA alloantibodies and autoantibodies, for which immune activity has been proven to favor allograft rejection in kidney and heart transplant. The development of anti- 
angiotensin II type I receptor and anti-endothelin-type A receptor antibodies (non-HLA antibodies) was investigated versus the clinical course and histopathologic findings in 29 ITx recipients. ${ }^{12}$ Twenty patients developed anti-angiotensin II type I receptor and / or anti-endothelin-type A receptor antibodies and 9 did not (control group). The non-HLA antibody groups had higher rates of allograft rejection than the control group (80\% vs 55\%) and significantly higher rates of antibody-mediated rejection $(55 \%$ vs $11 \%$; $P<.01)$ with detection of antiHLA DSAs. All rejection episodes in the non-HLA antibody groups appeared around the time of positive non-HLA antibody detection. Five patients had ACR at the time of non-HLA antibody development and 4 had viral infections. These results suggest that antibody-mediated mechanisms, targeting antigens beyond HLA, may trigger and accelerate immune responses and non-HLA antibodies could enhance rejection and affect long-term allograft survival.

\section{Expression of inflammatory and anti-inflammatory markers during chronic rejection}

From previously described reports, we could assume that CR after ITx is not only an immunologic mechanism of graft chronic damage (mediated by cell immunity, DSA, and non-HLA antibodies) but also a complex cell stress response involving numerous inflammatory and anti-inflammatory markers. In 2013, Wang and colleagues ${ }^{35}$ assessed the expression of 5 cell stress molecules during rat small bowel allograft CR (heat shock protein [HSP]-60, HSP70, interferon- $\alpha$, rat retinoic acid early inducible1-like transcript, and vasoactive intestinal peptide). A rat model of small bowel allograft rejection was induced on POD90 using Fisher 344 rats as donors and Lewis rats as recipients, which were immunosuppressed by cyclosporine. Lamina propria HSP60 (mean rank grade of 8.3 and 3.2, respectively), graft rat retinoic acid early inducible-1-like transcript (relative mRNA amounts of $5.1 \pm 1.8$ and $3.0 \pm 1.3$, respectively), and IFN- $\alpha$ (relative mRNA amounts of $108.3 \pm 49.2$ and $59.2 \pm 22.0$, respectively) expression levels were increased in the allograft group compared with the isograft group, suggesting the presence of cell stress during chronic small bowel allograft rejection. This cell response could ultimately provoke activation of natural killer cells and the subsequent downstream innate immune system. Natural killer cell activation has been found during the pathologic process of transplant from IRI to CR in other solid organs. Because natural killer cells are not targeted by current immunosuppressive therapies, including cyclosporine, this may explain why CR still developed under immunosuppressive treatment after ITx.

In another rat study, Gerlach and associates ${ }^{36}$ studied single-dose tacrolimus at varying concentrations to simulate the clinical situation of ACR versus moderate and severe CR. Graft histology and immune histology were assessed on POD7, POD14, and POD45. Intragraft and peripheral gene expression of inflammatory and anti-inflammatory markers, plasma lipopolysaccharide binding protein, and alloantibodies were simultaneously measured. In rats that received $1 \mathrm{mg}$ tacrolimus, acute rejection and recipient death occurred; however, $3 \mathrm{mg}$ and 5 $\mathrm{mg}$ prolonged survival and led to severe or moderate $\mathrm{CR}$, respectively, with $50 \%$ of the $5-\mathrm{mg}$ tacrolimus recipients surviving the observation period. Consequently, the authors observed severe infiltration on POD7 in untreated and in the 1-mg tacrolimus groups compared with minor histologic alterations in the 3-mg and 5-mg groups. Only treatment with $5 \mathrm{mg}$ tacrolimus prevented accumulation of CD4-positive $\mathrm{T}$ cells and ED1-positive macrophages during the observation period. Peripheral and intragraft expression levels of the T-cell activation inhibitormitochondrial gene, previously named Toag-1 (tolerance associated gene-1), were stable in the longterm surviving 5-mg group but declined before acute rejection or CR in the 1-mg and 3-mg groups. In contrast, lipopolysaccharide binding protein levels increased during acute rejection and CR. With this preclinical model, the authors were able to track different parameters during the course of rejection, identifying early, noninvasive markers that may help differentiate between long-term graft acceptance and graft rejection, thus potentially improving posttransplant monitoring and allowing for an early initiation of antirejection treatment.

\section{Magnitude of chronic rejection in clinical series}

In reviewing the incidence of $C R$ in clinical series, to quantify its present burden on ITx patients, it is worthwhile to start with the largest study from Pittsburgh. In 2012, Abu-Elmagd and associates ${ }^{14,37}$ observed CR in 36 allografts (19\%) of 194 patients with ITx (71 with liver) at 2 to 87 months posttransplant (mean of $21 \pm 10 \mathrm{mo}$ ). Concomitant active 
injury was observed in most biopsies. Each of the transplanted organs was affected, with the highest injury in the intestine. Graft failure due to CR was inevitable in 33 recipients $(92 \%)$. Cumulative risk of $\mathrm{CR}$ was modestly higher $(P>.05)$ in patients with positive versus negative crossmatch, with 5 -year incidences of $29 \pm 7$ and $21 \pm 4$, respectively. Utilizing DSA-free allografts as a control, the group showed that $\mathrm{CR}$ cumulative probability was significantly $(P<.001)$ higher in recipients with persistent and de novo DSA, independent $(P>.1)$ of visceral allograft type. Acute cellular rejection within 90 days was a significant risk factor $(P=.02)$ for $C R$, whereas type of immunosuppression was not a significant predictor $(P=.6)$. This relatively high incidence of CR of $19 \%$ in their series (compared with the previous $8 \%$ reported by the same group ${ }^{38}$ ) could be partially explained by the later strict immunosuppressive "weaning" protocol adopted by the Pittsburgh group.

In a pediatric study, Nayyar and associates ${ }^{39}$ reported CR rates of $>10 \%$ after ITx. A broader, single-center series showed an incidence of CR of $11 \%$; interestingly, the organ most affected by $\mathrm{CR}$ was the pancreas in the multivisceral graft. ${ }^{40}$ On the other hand, Gerlach and associates ${ }^{41}$ reported no incidence of CR in their series of 30 ITx recipients (18 isolated, 12 multivisceral). Their immunosuppression was mainly induced by thymoglobulin and infliximab and maintained by tacrolimus and mycophenolate mofetil/sirolimus. If patients produced DSAs, treatment with plasmapheresis and intravenous immunoglobulin was initiated. In the event of DSA persistence and/or treatment-refractory rejection, rituximab and/or bortezomib was added. Other clinical studies have shown CR rates after ITx ranging from $5 \%$ to $10 \%$. In an older study, ${ }^{42} 2$ of 28 recipients $(7.1 \%)$ developed CR. In 2011, the topic was widely addressed during the XII ISBTS meeting (unpublished results), with CR rates of $7 \%$ (8 recipients, $3.5 \pm 2.4$ y after ITx) reported by Farmer and associates from Los Angeles, CA, USA, 9\% (8 recipients, with half having combined liver-bowel transplant) reported by Gupte and colleagues from Birmingham, UK, 4.9\% (5 failed grafts) reported by Lacaille and associates from Paris, France, and 2\% reported by Matsumoto and colleagues from Washington, DC, USA. In 2016, another group from Buenos Aires, Argentina ${ }^{43}$ reported a $7.1 \%$ rate of graft loss due to CR (3 of 42 ITx, in which 32 were isolated and 10 were multivisceral). In another study $^{15}$ that included 79 intestine/multivisceral recipients (40 liver-inclusive allografts), CR affected 6 patients $(8 \%)$ and was numerically higher in patients with de novo DSA $(14 \%$ vs $5 \% ; P=.21)$. In a report of 48 adult recipients ( 49 grafts) treated from 2001 to 2011,445 recipients $(8 \%)$ received an isolated bowel graft and experienced CR, always preceded by ACR episodes. Two of these patients had survived after surgery (one after partial bowel resection). There was another case of segmental involvement of bowel by $C R$, with subsequent successful graft salvage by partial resection, as reported by Kim and associates, ${ }^{45}$ although this occurrence is rarely seen in the literature. In a study of 23 patients (24 ITx procedures, including 9 with isolated ITx, 11 with combined liver-ITx, and 4 with multivisceral ITx) from 1999 to 2104 from Ceulemans and associates, ${ }^{46}$ CR occurred in 2 patients $(8.3 \%)$.

The living-related ITx experience did not change the incidence of CR, with incidence of $10 \%(1 / 10$ patients) previously reported by one group. ${ }^{47}$ There were other reports at the XIII ISBTS 2013 (Oxford, UK) and the XIV ISBTS 2015 (Buenos Aires, Argentina) meetings, showing mean rates of between $5 \%$ and $10 \%$ (unpublished results). Table 1 summarizes the main results.

\begin{tabular}{|c|c|}
\hline Clinical Series & $\begin{array}{l}\text { Year of Report and Chronic } \\
\text { Rejection Rate, } \%\end{array}$ \\
\hline Pittsburgh, PA, USA $14,37,38$ & $2012=19 \% ; 2003=8 \%$ \\
\hline Chicago, IL, USA 47 & Living-related $2009=10 \%$ \\
\hline Birmingham, UK (unpublished results) & $2011=9 \%$ \\
\hline Belgium (5 centers) $)^{46}$ & $2015=8.3 \%$ \\
\hline Bologna, Italy 44 & $2013=8 \%$ \\
\hline Indianapolis, IN, USA 15 & $2015=8 \%$ \\
\hline Buenos Aires, Argentina ${ }^{43}$ & $2016=7.1 \%$ \\
\hline Omaha, NE, USA ${ }^{42}$ & $2002=7.1 \%$ \\
\hline Los Angeles, CA, USA (unpublished results) & $2011=7 \%$ \\
\hline Paris, France (unpublished results) & $2011=4.9 \%$ \\
\hline Washington, DC, USA (unpublished results) & $2011=2 \%$ \\
\hline Berlin, Germany ${ }^{41}$ & $2014=0 \%$ \\
\hline Leuven, Belgium²6 & $2016=0 \%$ \\
\hline Mean rate & $7.2 \%$ \\
\hline
\end{tabular}

\section{Discussion}

The advent of tacrolimus-based immunosuppression has facilitated dramatic improvements in short-term outcomes for ITx recipients. However, CR and chronic allograft enteropathy still represent one of the main causes of late graft loss. As with other solidorgan transplants, CR is affected and likely caused 
by an interaction of numerous nonimmune and immune factors. ${ }^{48,49}$ Apart from ACR prevention, here we have identified 3 factors that affect long-term allograft survival after ITx: the presence of inflammatory factors (ie, TNF- $\alpha$ and inflammatory and anti-inflammatory markers), humoral immunity activity (ie, DSAs and non-HLA antibodies), and the development of microchimerism and tolerance.

Chronic rejection starts with inflammation of the arteries, subsequently generating ischemic injuries to the graft because of obliterative arteriopathy, a typical feature described in heart, kidney, and lung recipients and also known as chronic transplant vasculopathy. ${ }^{50}$ Donor endothelial cells, expressing both type I and II MHC molecules (HLA), are attacked by mononuclear and polymorphonuclear infiltrates mediated by TNF- $\alpha$. When the recipient's immune system encounters donor endothelial cells, this interaction induces apoptosis of the endothelial cells themselves, generating hyperadhesiveness to leukocytes and recruitment of other leukocytes. This, in turn, induces a continuous fibroproliferative reaction. Because of chronic inflammation resulting from cellular infiltrates, organ adhesions occur, which are caused by fibrosis of the parenchyma. This theory is the so-called "direct injury hypothesis" 51 (Figure 1).

Humoral immunity affects graft survival, as shown by hyperacute rejection with necrosis of the allograft after reperfusion, a preformed antibody-

Figure 1. "Direct Injury Hypothesis" of Chronic Rejection

\section{DIRECT INIURY HYPOTHESIS}

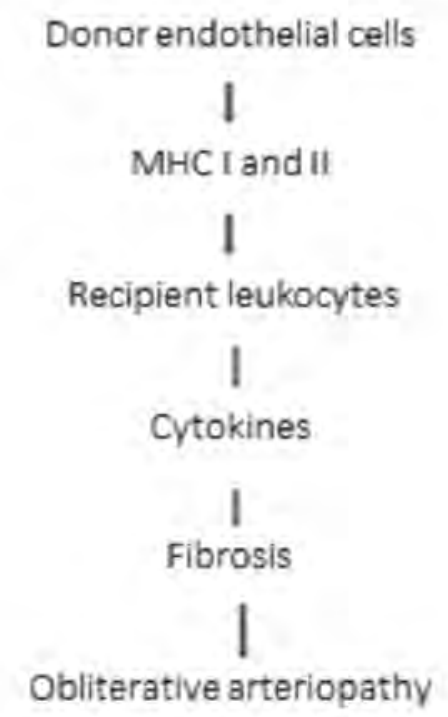

mediated process. However, the clear association with presensitization of the recipient allows the selection of patients at risk and few attempts at desensitization. Plasmapheresis and/or combined liver-ITx allows aggressive removal of DSAs, as DSAs negatively affect graft survival and the liver offers some degree of protection from preformed DSAs. ${ }^{52}$ Although individual case reports exist, desensitization protocols have not yet been applied in a systematic fashion. With the increasing number of recipients requiring retransplant, it is to be expected that high panel reactive antibody candidates will be increasingly common on ITx wait lists.

Microchimerism is the presence of a small number of cells that originate from another individual and are therefore genetically distinct from the cells of the host individual. Solid-organ transplant provokes chimerism through immune activation, thanks to the initial engagement of the donor and recipient lymphoid systems balanced by immunosuppression. This occurrence could be followed by a more stable relationship when immunosuppressants can be considerably lowered or even withdrawn. Previously, the presence of donor passenger leukocytes had been considered to be essentially deleterious to allograft survival, directly sensitizing the recipient's immune system. Destruction of these donor cells was thought to improve the donor/recipient immunologic interface. Later, the exact opposite was proven, in which it was shown that donor passenger leukocytes persisted in successful long-term allograft recipients but were destroyed in recipients with CR. This transplant form of microchimerism has shown that donor passenger leukocytes are necessary, but alone not sufficient, to induce tolerance and thus freedom from CR.

In their study of microchimerism in 1997, Demetris and colleagues ${ }^{51}$ created a historical, experimental ITx animal model of CR. They created 2 groups of genetically identical recipients: "CR-resistant" by pretreatment with a donor liver allograft and "CRprone" by bone marrow infusion. A heart allograft was implanted without immunosuppression in both groups, causing a transient ACR within days, but then the cardiac grafts were accepted and remained functional for more than 100 days. However, the group pretreated with a bone marrow infusion eventually developed CR and obliterative arteriopathy, whereas those pretreated with a liver became resistant to CR. The occurrence of ACR was overtaken easily by both 
groups, although with important differences. In the CR-prone animals, ACR was characterized by presence of recipient macrophages and their cytokines, upregulation of class II MHC antigens on the vascular endothelia, and loss of intragraft and extragraft and donor passenger leukocytes. In contrast, ACR in the CR-resistant group showed a weak endothelial class II MHC and macrophage expression, but the presence of transient and passenger leukocytes persisted in the graft and recipient lymphoid tissues. Thus, the major differences between these 2 groups were the persistence of donor passenger leukocytes in the CR-resistant animals and heightened recipient macrophage influx in the CR-prone group.

Induction of tolerance after ITx through establishment of mixed chimeras could be a potential approach to solve graft survival and toxicity associated with the use of immunosuppressants. Several recent studies demonstrated that Tregs could promote the establishment of allogeneic mixed chimerism and the induction of donor-specific tolerance. ${ }^{53}$ Regulatory T-cell-based therapy could prevent allograft rejection. In addition, Tregs not only play a critical role in controlling both ACR and CR but also might contribute to the protection of the allograft from IRI. ${ }^{53}$ A cotransplant of donor bone marrow plus Tregs specific for alloantigens could be an effective regimen to induce transplant tolerance, improving the overall outcomes of ITx.

\section{Conclusions}

There is an abundance of research over the past 20 years to clarify the mechanisms of CR development after ITx. Many animal models have simplified the complex steps leading toward chronic allograft enteropathy, highlighting the role of the different key players acting in a human scenario. About $5 \%$ to $10 \%$ of recipients worldwide are affected by $\mathrm{CR}$, but its early diagnosis remains difficult and pharmacologic options are still not available. Unfortunately, a graftectomy still represents the criterion standard for diagnosis and therapy. Despite these drawbacks, important steps have been undertaken toward full comprehension of this phenomenon. Although it remains important to prevent severe ACR episodes, the role of humoral immunity (and the way to prevent it) is gaining acceptance as one of the main factors related to CR development. In addition, nonimmune, inflammatory factors act as co-players in this complex process. The discovery of new immunosuppressants (as single drugs or in combination) could transform microchimerism and tolerance from chimeras to reality, enabling all recipients to prevent $\mathrm{CR}$. A multicenter approach is required to achieve this goal.

\section{References}

1. Todo S, Tzakis AG, Abu-Elmagd K, et al. Intestinal transplantation in composite visceral grafts or alone. Ann Surg. 1992;216(3):223234.

2. Abu-Elmagd KM, Tzakis A, Todo S, et al. Monitoring and treatment of intestinal allograft rejection in humans. Transplant Proc. 1993; 25(1 Pt 2):1202-1203

3. Abu-Elmagd K, Todo S, Tzakis A, et al. Rejection of human intestinal allografts: alone or in combination with the liver Transplant Proc. 1994;26(3):1430-1431.

4. Lee RG, Nakamura K, Tsamandas AC, et al. Pathology of human intestinal transplantation. Gastroenterology. 1996;110(6):18201834.

5. Grant D, Abu-Elmagd K, Mazariegos G, et al. Intestinal transplant registry report: global activity and trends. Am J Transplant. 2015;15(1):210-219.

6. Wu GS. Updates on antibody-mediated rejection in intestinal transplantation. World J Transplant. 2016;6(3):564-572.

7. Minneci PC. Intestinal transplantation: An overview. Pathophysiology. 2014;21(1):119-122.

8. Remotti H, Subramanian S, Martinez M, Kato T, Magid MS. Smallbowel allograft biopsies in the management of small-intestinal and multivisceral transplant recipients: histopathologic review and clinical correlations. Arch Pathol Lab Med. 2012;136(7):761-771.

9. Pech T, von Websky M, Ohsawa I, et al. Intestinal regeneration, residual function and immunological priming following rescue therapy after rat small bowel transplantation. Am J Transplant. 2012;12 Suppl 4:S9-S17.

10. de Bruin RW, Stein-Oakley AN, Kouwenhoven EA, et al. Functional, histological, and inflammatory changes in chronically rejecting small bowel transplants. Transpl Int. 2000;13(1):1-11.

11. Li Y, Zhu Y, Wang J, et al. Long-term comparison of rat model of chronic allograft rejection of orthotopic small bowel transplantation induced by cyclosporine versus tacrolimus. Transplant Proc. 2013;45(5):1811-1815.

12. Gerlach UA, Lachmann N, Ranucci G, et al. Non-HLA Antibodies may accelerate immune responses after intestinal and multivisceral transplantation. Transplantation. 2017:101(1):141149

13. Bond G, Reyes J, Mazariegos G, et al. The impact of positive T-cell lymphocytotoxic crossmatch on intestinal allograft rejection and survival. Transplant Proc. 2000;32(6):1197-1198.

14. Abu-Elmagd KM, Wu G, Costa G, et al. Preformed and de novo donor specific antibodies in visceral transplantation: long-term outcome with special reference to the liver. Am J Transplant. 2012;12(11):3047-3060.

15. Kubal C, Mangus R, Saxena R, et al. Prospective monitoring of donor-specific anti-HLA antibodies after intestine/multivisceral transplantation: significance of de novo antibodies. Transplantation. 2015;99(8):e49-e56.

16. Gerlach UA, Schoenemann C, Lachmann N, Koch M, Pascher A Salvage therapy for refractory rejection and persistence of donorspecific antibodies after intestinal transplantation using the proteasome inhibitor bortezomib. Transp/ Int. 2011;24(5):e43-e45.

17. Meyer D, Otto C, Rummel C, et al. "Tolerogenic effect" of the liver for a small bowel allograft. Transpl Int. 2000;13 Suppl 1:S123S126.

18. Wu G, Cruz RJ. Liver inclusion improves outcomes of intestinal retransplantation in adults. [Corrected]. Transplantation. 2015; 99(6):1265-1272. 
19. Tryphonopoulos P, Weppler D, Nishida S, et al. Mucosal fibrosis in intestinal transplant biopsies correlates positively with the development of chronic rejection. Transplant Proc. 2006;38(6): 1685-1686.

20. Swanson BJ, Talmon GA, Wisecarver JW, Grant WJ, Radio SJ. Histologic analysis of chronic rejection in small bowel transplantation: mucosal and vascular alterations. Transplantation. 2013:95(2):378-382.

21. Kouwenhoven EA, Stein-Oakley AN, Maguire JA, et al. Increased expression of basic fibroblast growth factor during chronic rejection in intestinal transplants is associated with macrophage infiltrates. Transpl Int. 1999;12(1):42-49.

22. Orloff $\mathrm{SL}$, Yin $\mathrm{Q}$, Corless $\mathrm{CL}$, et al. Tolerance induced by bone marrow chimerism prevents transplant vascular sclerosis in a rat model of small bowel transplant chronic rejection. Transplantation. 2000;69(7):1295-1303.

23. Guo Z, Wang J, Dong Y, et al. Long-term survival of intestinal allografts induced by costimulation blockade, busulfan and donor bone marrow infusion. Am J Transplant. 2003:3(9):1091-1098.

24. Murase $N$, Ye $Q$, Nalesnik MA, et al. Immunomodulation for intestinal transplantation by allograft irradiation, adjunct donor bone marrow infusion, or both. Transplantation. 2000;70(11):16321641.

25. Timmermann W, Otto C, Gasser M, et al. Long-term small bowel allograft function induced by short-term FK 506 application is associated with split tolerance. Transp/ Int. 2000;13 Suppl 1:S532S536.

26. Ceulemans LJ, Braza F, Monbaliu D, et al. The Leuven Immunomodulatory Protocol promotes T-regulatory cells and substantially prolongs survival after first intestinal transplantation. Am J Transplant. 2016;16(10):2973-2985.

27. Wang J, Li Y, Li J. Attenuation of rat chronic small bowel allograft rejection by $\mathrm{n}-3$ polyunsaturated fatty acids is associated with reduced expression of graft IL-15. Pediatr Transplant. 2013;17(6): 561-566.

28. Li Q, Zhang Q, Wang C, et al. Fish oil enhances recovery of intestinal microbiota and epithelial integrity in chronic rejection of intestinal transplant. PLoS One. 2011;6(6):e20460.

29. Wei W, Zhu Y, Wang J, et al. Six-month feeding of low-dose fish oil decreases vascular expression of high mobility group box 1 and receptor for advanced glycation end-products in rat chronic allograft vasculopathy. Transplant Proc. 2013:45(5):1771-1775.

30. Wei W, Chen M, Zhu Y, et al. Down-regulation of vascular HMGB1 and RAGE expression by $n-3$ polyunsaturated fatty acids is accompanied by amelioration of chronic vasculopathy of small bowel allografts. J Nutr Biochem. 2012;23(10):1333-1340.

31. Matsumoto CS, Kaufman SS, Island ER, et al. Hepatic explant pathology of pediatric intestinal transplant recipients previously treated with omega-3 fatty acid lipid emulsion. J Pediatr. 2014;165(1):59-64.

32. Langrehr JM, Gube K, Hammer MH, et al. Short-term anti-CD4 plus anti-TNF-alpha receptor treatment in allogeneic small bowel transplantation results in long-term survival. Transplantation. 2007:84(5):639-646.

33. Gerlach UA, Koch M, Muller HP, et al. Tumor necrosis factor alpha inhibitors as immunomodulatory antirejection agents after intestinal transplantation. Am J Transplant. 2011;11(5):1041-1050.

34. Gerlach UA, Atanasov G, Wallenta L, et al. Short-term TNF-alpha inhibition reduces short-term and long-term inflammatory changes post-ischemia/reperfusion in rat intestinal transplantation. Transplantation. 2014;97(7):732-739.
35. Wang J, Li Y, Li J. Cell stress response in rat chronic small bowel allograft rejection. Transplant Proc. 2013;45(6):2539-2542.

36. Gerlach UA, Klopfel M, Atanasov G, et al. Intragraft and systemic immune parameters discriminating between rejection and longterm graft function in a preclinical model of intestinal transplantation. Transplantation. 2017:101(5):1036-1045.

37. Abu-Elmagd KM, Costa G, Bond GJ, et al. A decade of experience with a single dose of rabbit antithymocyte globulin or alemtuzumab pretreatment for intestinal and multivisceral transplantation. Clin Transpl. 2012:155-166.

38. Parizhskaya M, Redondo C, Demetris A, et al. Chronic rejection of small bowel grafts: pediatric and adult study of risk factors and morphologic progression. Pediatr Dev Pathol. 2003;6(3):240-250.

39. Nayyar N, Mazariegos G, Ranganathan S, et al. Pediatric small bowel transplantation. Semin Pediatr Surg. 2010;19(1):68-77.

40. Takahashi H, Kato T, DelacruzV, et al. Analysis of acute and chronic rejection in multiple organ allografts from retransplantation and autopsy cases of multivisceral transplantation. Transplantation. 2008;85(11):1610-1616.

41. Gerlach UA, Lachmann N, Sawitzki B, et al. Clinical relevance of the de novo production of anti-HLA antibodies following intestinal and multivisceral transplantation. Transp/ Int. 2014;27(3): 280-289.

42. Iyer KR, Srinath C, Horslen S, et al. Late graft loss and long-term outcome after isolated intestinal transplantation in children. $J$ Pediatr Surg. 2002;37(2):151-154

43. Ramisch D, Rumbo C, Echevarria C, et al. Long-term outcomes of intestinal and multivisceral transplantation at a single center in Argentina. Transplant Proc. 2016;48(2):457-462.

44. Lauro A, Bagni A, Zanfi C, et al. Mortality after steroid-resistant acute cellular rejection and chronic rejection episodes in adult intestinal transplants: report from a single center in induction/preconditioning era. Transplant Proc. 2013;45(5):20322033.

45. Kim SY, Kim DG, Moon IS, et al. Chronic rejection in a small bowel transplant with successful revision of the allograft by segmental resection: case report. Transplant Proc. 2012;44(4):1180-1182.

46. Ceulemans $L$, Monbaliu D, De Roover A, et al. Belgian multicenter experience with intestinal transplantation. Transpl Int. 2015; 28(12):1362-1370.

47. Gangemi A, Tzvetanov IG, Beatty E, et al. Lessons learned in pediatric small bowel and liver transplantation from living-related donors. Transplantation. 2009:87(7):1027-1030.

48. Ruiz P. How can pathologists help to diagnose late complications in small bowel and multivisceral transplantation? Curr Opin Organ Transplant. 2012;17(3):273-279.

49. Ruiz P. Updates on acute and chronic rejection in small bowel and multivisceral allografts. Curr Opin Organ Transplant. 2014;19(3): 293-302.

50. Trentadue G, Dijkstra G. Current understanding of alloimmunity of the intestinal graft. Curr Opin Organ Transplant. 2015;20(3):286294.

51. Demetris AJ, Murase N, Lee RG, et al. Chronic rejection. A general overview of histopathology and pathophysiology with emphasis on liver, heart and intestinal allografts. Ann Transplant. 1997;2(2): 27-44.

52. Dick AA, Horslen S. Antibody-mediated rejection after intestinal transplantation. Curr Opin Organ Transplant. 2012;17(3):250-257.

53. Du JF, Li SY, Yu B, Bai X. Treg-based therapy and mixed chimerism in small intestinal transplantation: does Treg +BMT equal intestine allograft tolerance? Med Hypotheses. 2011; 76(1):77-78. 